\title{
Controllability of transportation networks
}

\author{
Marco Rinaldi, Ph.D., University of Luxembourg \\ Francesco Viti, Ph.D., University of Luxembourg
}

\section{Introduction}

Researchers in the past decades have been developing advanced dynamic traffic management policies that i) are based on coordination of individual control points and ii) explicitly take into account the network-wide effects of control actions, thanks to predictive traffic propagation and route choice modelling. In works such as those of (Aboudolas et al., 2010; Geroliminis and Daganzo, 2008; Rinaldi et al., 2016; Rinaldi and Tampère, 2015; Taale, 2008; Taale and Hoogendoorn, 2012) network-wide approaches combined with model-based control strategies have been object of considerable research effort.

While the body of literature concerning traffic control strategies evolved considerably over the years, little attention has been devoted to strategic network control design: determining the location, kind and amount of controllers contributing to the aforementioned coordinated strategies is largely an adhoc process, in which either existing infrastructure is simply adapted and connected, or expert knowledge is applied in a case-by-case fashion, disregarding the role that control infrastructure plays in network-wide performances (Cantarella and Sforza, 1995).

Missing a network design view of controller placement can be considerably detrimental: the maximum extent to which any control strategy can steer traffic conditions towards more optimal alternatives is operationally bound to only those flow distributions that can be triggered by the existing set of controllers.

In this work, we explore the relationship between location and amount of controllers and the corresponding network-wide performance, showcasing how indeed these topological attributes are directly linked to the network's emergent behaviour. We exploit the control theoretical concept of structural controllability of complex networks (Lin, 1974; Liu et al., 2011) to relate the topological aspects of the equipped control intrastructure and the resulting network dynamics, and perform validation on a simple case study, showcasing how indeed full controllability solutions are capable of reaching System Optimal performances.

\section{Methodology}

In order to apply the theory of structural controllability of complex networks to the case of transportation networks, after selecting as descriptive state of the system the Cumulative Vehicles Number at nodes (from which all other significative measures of traffic performance can easily be derived), we assume the following:

- Linearity: the dynamics of the Cumulative Vehicle Number at nodes are slow enough to be correctly represented by piecewise affine functions;

- Newell's Kinematic Wave Theory compliant flow propagation dynamics;

- Triangular Fundamental Diagram;

- Deterministic User Equilibrium route choice: users entering the network have perfect information of the network travel times they will experience, and react accordingly in terms of chosen routes; 
- The effects of traffic control policies are fully captured by the input matrix elements $\left\{b_{i j}\right\}$ and input signal vector $U(\cdot)$;

- Travel times between consecutive nodes (including both topological effects and congestion effects) are fully captured by the state matrix elements $\left\{a_{i j}\right\}$.

Based upon these assumptions, we develop the formulation of Equation (1) (for the sake of concision, the full derivation is left out of the abstract):

$$
\begin{aligned}
& X(k)=A \cdot X(k-1)+B \cdot U(k-1) \\
& A=\left\{a_{i j}\right\} \in \mathfrak{R}^{n \times n} \\
& B=\left\{b_{i j}\right\} \in \mathfrak{R}^{n \times m} \\
& m=|G|+|\Phi| \\
& b_{i j}=\left\{\begin{array}{l}
1 \forall j: g_{j}>0 \\
1 / 2 \forall(i, j): \exists h \in N: \phi_{i j}+\phi_{h j}=1 \\
0 \text { otherwise }
\end{array}\right. \\
& u_{j}(k)=\left\{\begin{array}{l}
-\xi_{j}(k-1) x_{i}(k-1) \quad \forall(i, j): g_{j}>0 \\
a_{i j} x_{j}(k-1) \cdot\left(1-\phi_{i j}(k-1)\right)+ \\
+a_{h j} x_{j}(k-1) \cdot\left(\phi_{i j}(k-1)-1\right) \forall j: \exists(i, j, h) \in N: \phi_{i j}+\phi_{h j}=1 \\
0 \text { otherwise }
\end{array}\right.
\end{aligned}
$$

where $n=|N|$ is the total amount of nodes in the network, $G=\left\{g_{j}\right\}$ is the set of pricing controllers equipped on the network and $\Phi=\left\{\phi_{i j}\right\}$ that of traffic light controllers. The structure of matrix $A$, i.e. knowledge on which elements $\left\{a_{i j}\right\}$ are equal to zero and which aren't, is assumed fixed and correct. The exact values, for the sake of our goals, are irrelevant, as long as the individual elements don't collapse to zero, which would directly impact the controllability properties of the given system. In network behaviour terms, this implies that we don't require exact information on how fast vehicles are traveling from a node to its successor(s), but only knowledge on whether or not any flow is present. Incidental situations in which said flows would become zero (gridlock conditions) are considered irrelevant from the perspective of designing controllers.

Following Kalman's controllability theory, once the network's dynamics are correctly modelled in the form (1), a network can be deemed controllable if the following condition holds:

$$
\begin{aligned}
& W_{c}=\left[\begin{array}{lllll}
B & A B & A^{2} B & \cdots & A^{n-1} B
\end{array}\right] \\
& r k\left(W_{c}\right)=n
\end{aligned}
$$

that is, the chosen controller set $M=G \cup \Phi$ yields full controllability if and only if the matrix rank of the controllability gramian $W_{c}$ is full.

In the following we show how indeed this condition holds for a simple network, and how for instances of control infrastructure yielding less than full rank, System Optimum cannot be reached. Further results on more general networks, derived through optimization, will be showcased at the symposium. 


\section{Results}

We perform a fully explorative analysis of the Total Cost objective function solution space, and how this varies for different sets of equipped controllers, on a very simple network, which we abundantly explored in recent works (Rinaldi et al., 2017), shown in Figure 1.

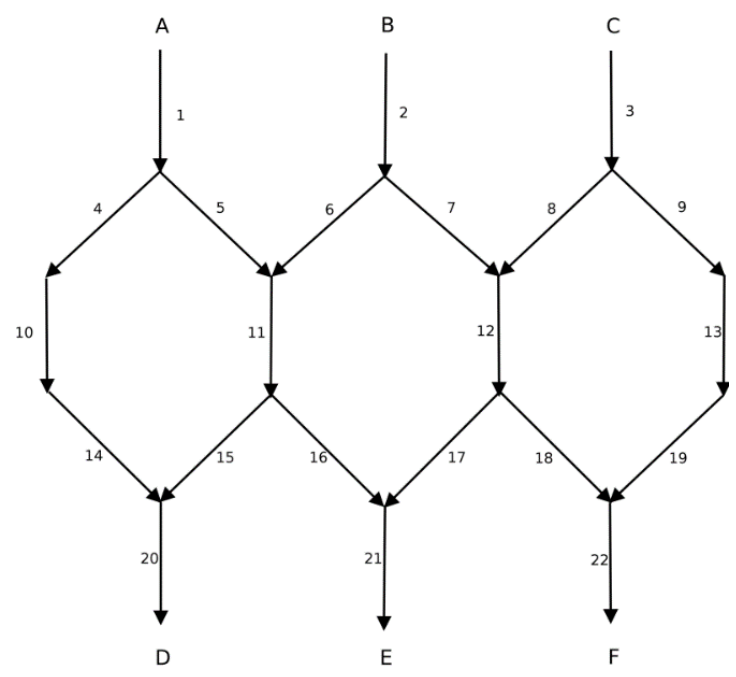

Figure 1: Three-PAS network.

We consider OD demand flowing vertically along couples A-D, B-E and C-F, and we assume that link cost functions $c_{l}\left(f_{l}\right)$ are constant for all links apart from links 10-13, who instead are equipped with the BPR cost function $c_{l}\left(f_{l}\right)=1+\alpha_{l} \cdot\left(f_{l} / 400\right)^{\beta}+g_{l}$, with the following parameter values:

Table 1: BPR function parameters for Test Case 1.

\begin{tabular}{ll}
\hline Link & Parameters \\
\hline Link 10 & $\alpha_{10}=0.5, \beta_{10}=2$ \\
Link 11 & $\alpha_{11}=0.2, \beta_{11}=4$ \\
Link 12 & $\alpha_{12}=0.5, \beta_{12}=4$ \\
Link 13 & $\alpha_{13}=0.4, \beta_{13}=2$
\end{tabular}

and where $g_{l}$ is the additional cost levied by a pricing controller, if installed on the given link.

We begin this exploration by examining the Total Cost objective function's shape when a single pricing controller is equipped on either link 10, 11, 12 or 13, as shown in Figure 2. 


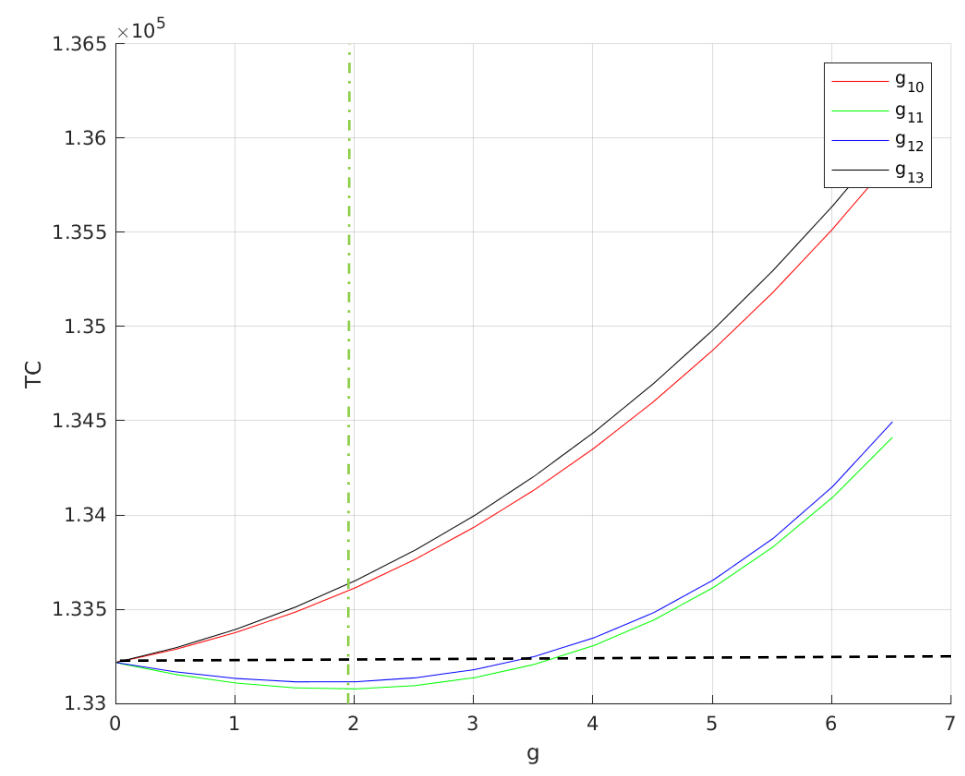

Figure 2: Total Cost objective function shapes for separate controllers.

Interestingly, equipping controllers $g_{10}$ or $g_{13}$ alone brings no gain to the system as a whole: the global minimum for both resulting objective functions is at $g^{*}=0$, thus yielding Total Cost equal, by definition, to the User Equilibrium value (marked in Figure 2 by the black dashed line). Controllers $g_{11}$ and $g_{12}$ instead both exhibit individual global minima in the vicinity of $g^{*}=2$ (marked in Figure 7 by the light green dashed line), bearing a visible reduction of Total Cost. For these four individual controllers, the percental distance from System Optimum and the corresponding degree of controllability (computed as $\theta(B)=r k\left(W_{c}(B)\right) / n$ ) are as follows:

$$
\begin{array}{ll}
\Delta T C_{g_{10}}=0.2606 \% & \theta\left(B_{g_{10}}\right)=1 / 3 \\
\Delta T C_{g_{11}}=0.1543 \% & \theta\left(B_{g_{11}}\right)=1 / 3 \\
\Delta T C_{g_{12}}=0.1829 \% & \theta\left(B_{g_{12}}\right)=1 / 3 \\
\Delta T C_{g_{13}}=0.2607 \% & \theta\left(B_{g_{13}}\right)=1 / 3
\end{array}
$$

We then consider all six possible couples of controllers, $\left[g_{10}, g_{11}\right],\left[g_{10}, g_{12}\right],\left[g_{10}, g_{13}\right],\left[g_{11}, g_{12}\right]$, $\left[g_{11}, g_{12}\right],\left[g_{12}, g_{13}\right]$, and show the respective Total Cost solution spaces in terms of contours in Figure 3(a-f).

The globally minimal points are easily identified by darker coloring, and are consistently found in the range $g<2.5$. While not very clear to the naked eye, within these six combinations a clear "best case" arises, as can be seen when considering the percental distance from System Optimal total cost:

$$
\begin{array}{ll}
\Delta T C_{g_{10}, g_{11}}=0.1548 \% & \Delta T C_{g_{10}, g_{12}}=0.1835 \% \\
\Delta T C_{g_{10}, g_{13}}=0.2616 \% & \Delta T C_{g_{11}, g_{12}}=0.006 \% \\
\Delta T C_{g_{11}, g_{13}}=0.1548 \% & \Delta T C_{g_{12}, g_{13}}=0.1835 \%
\end{array}
$$




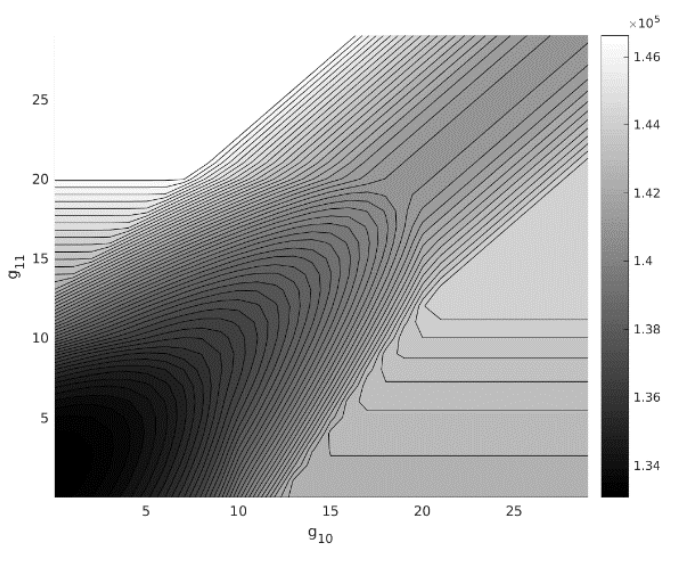

(a): $\left[g_{10}, g_{11}\right]$

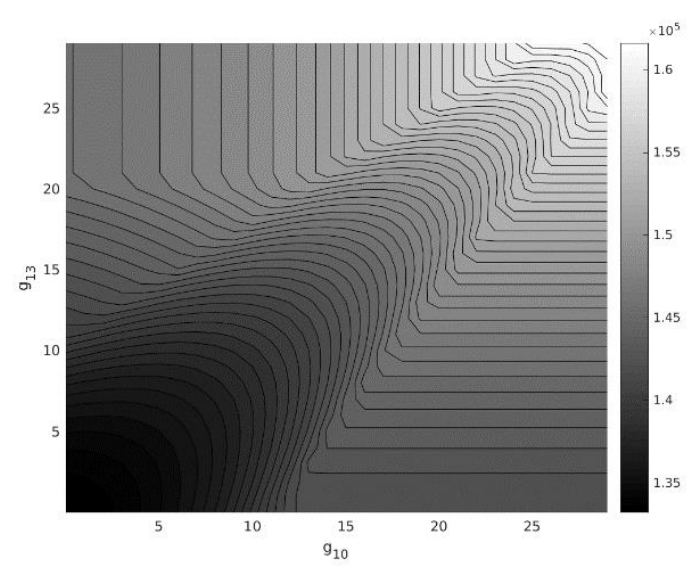

(c): $\left[g_{10}, g_{13}\right]$

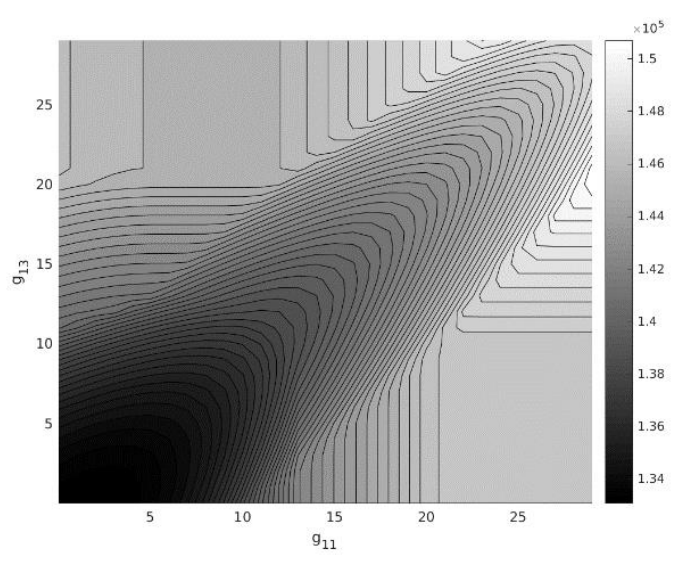

(e): $\left[g_{11}, g_{13}\right]$

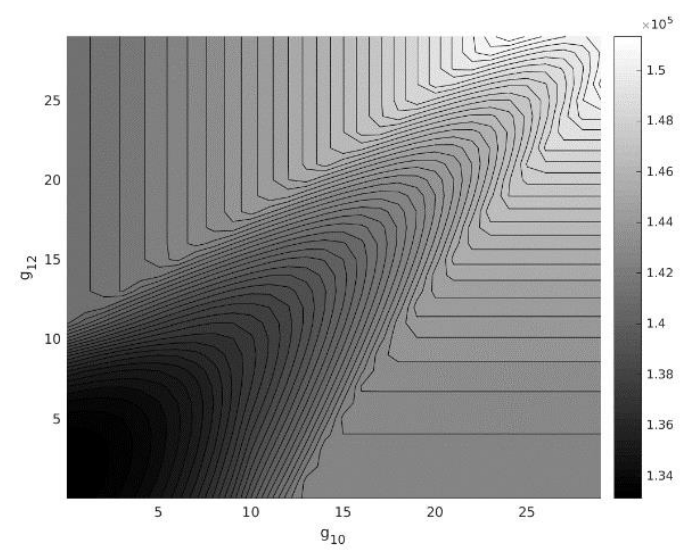

(b): $\left[g_{10}, g_{12}\right]$

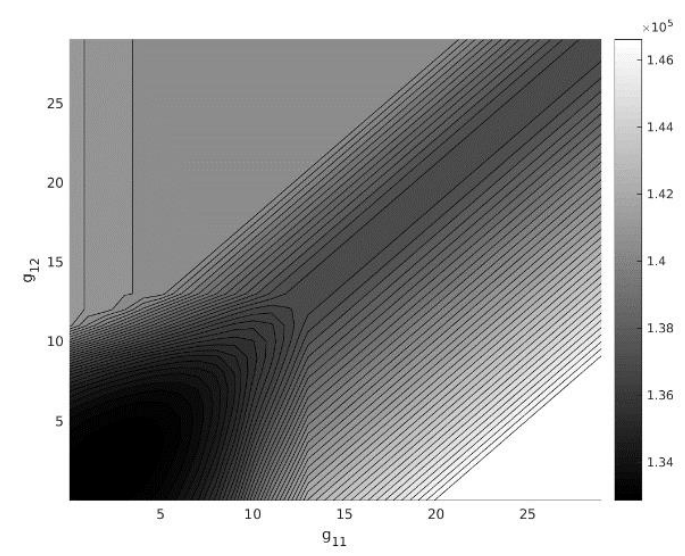

(d): $\left[g_{11}, g_{12}\right]$

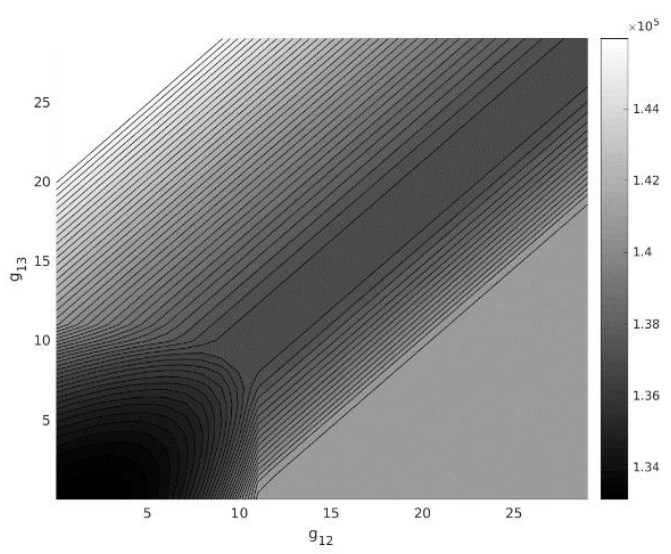

(f): $\left[g_{12}, g_{13}\right]$

Figure 3: Total Cost objective function solution space shape for different couples of controllers. 
The degree of controllability is again constant for all combinations, valued at $\theta(B)=2 / 3$. This is unsurprising: even though combination $\left[g_{11}, g_{12}\right]$ is closest to the System Optimal value, for this network no less than three controllers are necessary to achieve full controllability. Indeed, any combination of three controllers yields a value $\theta(B)=1$, and correctly captures the full threedimensional behaviour of this network. Equipping controllers beyond this amount, up to and including first best pricing solutions, yields no additional gains in terms of reachable network-wide performance.

\section{Conclusions}

In this work we investigated the extent to which choosing locations, kinds and amounts of controllers to be installed in a transportation network affect the performances of network-wide control approaches. Test results showed how indeed amount and locations of controllers influence networkwide performances, in terms of solution space shape and relevance of reachable minima with respect to System Optimum. Measuring the degree of controllability through our proposed formulation correctly captures this aspect.

During the symposium we will showcase how the degree of controllability can be exploited to develop a controller placement algorithm that parsimoniously locates controllers on general networks, and how this algorithm performs compared to other location strategies in literature.

\section{References}

Aboudolas, K., Papageorgiou, M., Kouvelas, A., Kosmatopoulos, E., 2010. A rolling-horizon quadratic-programming approach to the signal control problem in large-scale congested urban road networks. Transp. Res. Part C Emerg. Technol. 18, 680-694. https://doi.org/10.1016/j.trc.2009.06.003

Cantarella, G.E., Sforza, A., 1995. Network Design Models and Methods for Urban Traffic Management, in: Urban Traffic Networks, Transportation Analysis. Springer, Berlin, Heidelberg, pp. 123-153. https://doi.org/10.1007/978-3-642-79641-8_5

Geroliminis, N., Daganzo, C.F., 2008. Existence of urban-scale macroscopic fundamental diagrams: Some experimental findings. Transp. Res. Part B Methodol. 42, 759-770. https://doi.org/10.1016/j.trb.2008.02.002

Lin, C.-T., 1974. Structural controllability. IEEE Trans. Autom. Control 19, 201-208. https://doi.org/10.1109/TAC.1974.1100557

Liu, Y.-Y., Slotine, J.-J., Barabási, A.-L., 2011. Controllability of complex networks. Nature 473, 167-173. https://doi.org/10.1038/nature10011

Rinaldi, M., Himpe, W., Tampère, C., 2016. A sensitivity based approach for adaptive decomposition of anticipatory network traffic control. Transp. Res. C Emerg. Technol. https://doi.org/10.1016/j.trc.2016.01.005

Rinaldi, M., Tampère, C.M.J., 2015. An extended coordinate descent method for distributed anticipatory network traffic control. Transp. Res. Part B Methodol. 80, 107-131. https://doi.org/10.1016/j.trb.2015.06.017

Rinaldi, M., Tampère, C.M.J., Viti, F., 2017. On characterizing the relationship between route choice behaviour and optimal traffic control solution space. Transp. Res. Part B Methodol. https://doi.org/10.1016/j.trb.2017.08.014

Taale, H., 2008. Integrated Anticipatory Control of Road Networks. A Game-Theoretical Approach.

Taale, H., Hoogendoorn, S., 2012. Network-wide Traffic Management with Integrated Anticipatory Control. Presented at the Transportation Research Board 91st Annual Meeting. 